\title{
Expression of Endoplasmic Reticulum Stress Related Genes in Blood Cells of Obese Boys with and without Insulin Resistance
}

\author{
Dmytro O. Minchenko, $\mathrm{PhD}^{1,2}$ \\ ${ }^{1}$ Palladin Institute of Biochemistry National Academy of Sciences of Ukraine \\ ${ }^{2}$ O.O. Bohomolets National Medical University \\ Kyiv, Ukraine
}

\begin{abstract}
Objective: to study the changes in the expression level of the subset of genes, encoding for important cell growth factors and enzymes, which play an important role in the control of cellular growth and apoptosis, in blood cells of obese boys with and without insulin resistance for evaluation of its possible significance in the development of obesity and impaired insulin sensitivity.

Material and Methods: We studied the expression of genes, which are responsible for control of cell growth and survival, in blood cells of obese boys with normal and impaired insulin sensitivity as compared to normal (control) individuals.

Results: It was shown that the expression level of PLAGL, CYR61, ITGA5, and TFPI2 genes is increased, but the GADD45A gene is decreased in blood cells of obese children with normal insulin sensitivity as compared to the control group. Insulin resistance in obese boys leads to the up-regulation of PPP1R15A and PLAGL1 gene expressions as well as to the down-regulation of TFPI2, GADD45A, ALDH1A2, CYR61, and HSPA6 genes in blood cells as compared to obese patients with normal insulin sensitivity.

Conclusion: Results of this study provide evidence that obesity affects the expression of the subset of genes related to the control of cell growth and survival in blood cells and that impaired insulin sensitivity in obesity is associated with changes in the expression level of PLAGL, CYR61, GADD45A, PPP1R15A, TFPI2, ALDH1A2, and HSPA6 genes, which possibly contributes to the development of obesity and its metabolic complications, including insulin resistance.
\end{abstract}

Keywords: mRNA expression; CYR61; PLAGL; ITGA5; PPP1R15A; GADD45A; TFPI2; blood; obesity; insulin resistance.

\section{Introduction}

The development of obesity and its metabolic complications, one of the most profound public health problems, is associated with dysregulation of various intrinsic mechanisms, which control the most basic metabolic processes, as well as changes in numerous gene expressions, which contribute to the development of obesity as well as its metabolic complications and possibly reflect some changes in fat and other tissues. Obesity and its metabolic complications are associated with dysregulation of numerous intrinsic mechanisms, which control most of the key metabolic processes, including cellular growth, apoptosis, and insulin sensitivity [1-5]. Moreover, obesity and metabolic syndrome

*Corresponding author: Prof. Dmytro O. Minchenko, PhD, Palladin Institute of Biochemistry, Kyiv, Ukraine; e-mail: ominchenko@, yahoo.com result from interactions between genes and environmental factors and are associated with changes in gene expressions of the regulatory network in adipose tissue as well as in various organs and tissues, including blood cells [5-9]. Adipose tissue growth is a center of obesity, is tightly associated with apoptosis and the cell proliferation processes, and is controlled by different interconnected regulatory factors and enzymes $[1,6,8]$. Special interest should be given to key regulatory factors and enzymes, which control cell growth and survival, especially GADD45A, PLAGL1, CYR61, PPP1R15A, TFPI2, HSPA6, and ITGA5 [10-17].

The cysteine-rich angiogenic inducer 61 (CYR61), also known as CCN family member 1 and insulin-like growth factor-binding protein 10 (IGFBP10), is a secreted, cysteinerich, heparin-binding protein encoded by a growth factorinducible, immediate-early gene and acting as an extracellular, matrix-associated signaling molecule, which promotes matrix remodeling, cell proliferation, cell migration and adhesion by up-regulating the expression of a number of genes [10-12]. 
Moreover, the expression of the CYR61 gene is regulated by TP53, miRNA-22 and miRNA-100 as well as through activation of the PI3K/Akt signaling $[10,18,19]$. It is interesting to note that degradome products of this matricellular protein may modulate the pathological angiogenesis in the retina [20]. The PLAGL1 (pleiomorphic adenoma gene-like 1) gene encodes a $\mathrm{C} 2 \mathrm{H} 2$ zinc finger protein, which is a transcriptional regulator with anti-proliferative properties [21,22].

The ITGA5 (integrin, alpha 5) gene encodes an alpha polypeptide of fibronectin receptor, which interacts with different extracellular matrix proteins, including CYR61 and vascular endothelial growth factor receptor 2, and participates in cell-surface mediated signaling. Integrins can also activate protein kinases involved in the regulation of cell growth, division, survival, differentiation, migration and apoptosis $[13,23]$. There are data that TFPI2 (tissue factor pathway inhibitor 2) can also participate in the control of tumor growth preferentially through inhibition of a variety of serine proteases as well as through the regulation of plasmin-mediated matrix remodeling $[14,24,25]$. The growth arrest and DNA-damageinducible, alpha (GADD45A) gene plays an important role in cell cycle control as a regulator of some protein kinases and responds to environmental stresses $[15,26]$. Furthermore, GADD45A is associated with growth arrest and apoptosis and has both tumor suppressor and tumor promoter functions, depending on the tissue/cell type and transforming event $[15,26]$. PPP1R15A (protein phosphatase 1, regulatory subunit $15 \mathrm{~A})$ is a regulatory subunit of serine/threonine-protein phosphatase PP1, which dephosphorylates the translation initiation factor eIF-2A/EIF2S1, thereby reversing the shutoff of protein synthesis initiated by stress-inducible kinases and facilitating recovery of cells from stress, as well as downregulating the TGF-beta signaling pathway, and may promote apoptosis by inducing TP53 phosphorylation [16,27]. The aldehyde dehydrogenase 1 family member A2 (ALDH1A2) catalyzes the PPAR $\gamma$-directed synthesis of retinoic acid, the active derivative of vitamin A, which is a hormonal signaling molecule that functions in developing and adult tissues and has a relation to suppression of tumor growth $[17,28]$. The heat shock $70 \mathrm{kDa}$ protein- 6 is a stress responsible protein, which mediates the folding of newly translated polypeptides and is associated with childhood leukemia [29].

The endoplasmic reticulum stress is also recognized as an important determinant of obesity, insulin resistance, and impaired glucose tolerance and contributes to the expression profile of many regulatory genes, including GADD45A, PLAGL1, CYR61, PPPIR15A, TFPI2, HSPA6, and ITGA5, resulting in peripheral insulin resistance and other obesity complications [1,5,30-32], although detailed molecular mechanisms cannot be ruled out.

It is possible that identification of real mechanisms of metabolic abnormalities in obesity, as well as its complications at molecular and cellular levels, helps to better understand why obesity develops and why only some obese individuals develop secondary metabolic disorders [33]. However, a detailed molecular mechanism of the involvement of different genes of the regulatory network in the development of obesity and its complications is not clear yet and remains to be determined.
The main goal of this study was to clarify the role of the subset of gene expressions, encoding for important cell growth factors and enzymes, which play an important role in the control of cellular growth and apoptosis, in blood cells of obese boys with and without insulin resistance for evaluation of its possible significance in the development of obesity and impaired insulin sensitivity.

\section{Materials and Methods}

Fifteen boys participated in this study. They were divided into three equal groups (5 subjects in each group): normal individuals as control, obese patients with insulin resistance, and obese patients without insulin resistance. Written informed consent was obtained from all participants. The study was approved by the local research ethics committees of Institute of Children and Adolescent Health Care of the National Academy of Medical Science of Ukraine.

Clinical characteristics of the study participants are shown in Table 1. The normal (control) participants were individuals with mean age $14 \pm 0.7$ years and mean body mass index (BMI) $18.7 \pm 0.12 \mathrm{~kg} / \mathrm{m}^{2}$. The obese participants, both with normal insulin sensitivity and with insulin resistance, had a mean age of $14 \pm 0.6$ and $14 \pm 0.4$ years, respectively, and mean BMI $31.0 \pm 0.40$ and $34.2 \pm 2.39 \mathrm{~kg} / \mathrm{m}^{2}$, respectively.

Table 1.

Clinical characteristics of the study participants

\begin{tabular}{|l|c|c|c|}
\hline Variable & Control & Obesity & Obesity + IR \\
\hline Age at visit (years) & $14 \pm 0.73$ & $14 \pm 0.6$ & $14 \pm 0.38$ \\
\hline BMI $\left(\mathrm{kg} / \mathrm{m}^{2}\right)$ & $18.7 \pm 0.12$ & $31 \pm 0.40 *$ & $34.2 \pm 2.39 *$ \\
\hline HOMA-IR & $2.36 \pm 0.17$ & $2.70 \pm 0.28$ & $8.70 \pm 1.41 * \wedge$ \\
\hline FI $(\mathrm{mIU} / \mathrm{mL})$ & $13.0 \pm 0.95$ & $14.1 \pm 1.35$ & $43.4 \pm 6.70 * \wedge$ \\
\hline
\end{tabular}

$I R$ - insulin resistance; HOMA-IR - insulin resistance index; FI - fasting insulin; ${ }^{*}-P<0.05$ versus the control group; ${ }^{\wedge}{ }^{\wedge} P<0.05$ versus the obese group.

Thus, BMI, which is a main criterion of obesity, in these last two groups of patients was significantly higher $(+66$ and $+83 \%$, respectively; $\mathrm{P}<0.05$ in both cases) as compared to the control individuals (Table 1). Moreover, no significant changes were found in insulin resistance index in obese individuals as compared to the control group. In obese patients with impaired insulin sensitivity, versus both the control boys and the obese subjects with normal insulin sensitivity, the insulin resistance index was significantly increased (3.7 and 3.2 fold, respectively; $\mathrm{P}<0.05$ in both cases). Similar results were observed in the fasting insulin levels: no significant changes in obese individuals and strong increase in obese children with insulin resistance ( 3.3 fold; $\mathrm{P}<0.05$ ) as compared to the control group.

\section{$\underline{\text { RNA isolation }}$}

Trisol reagent (Invitrogen, USA) was used for RNA extraction from blood of normal (control) and obese individuals with or without insulin resistance. 
Reverse transcription and quantitative real-time polymerase chain reaction analysis

The expression levels of genes related to regulation of cell growth and glucose homeostasis (PLAGL, CYR61, GADD45A, GADD34(PPP1R15A), ITGA5, TFPI2, ALDH1A2, and HSPA6) were measured in blood cells by a real-time quantitative PCR of complementary DNA (cDNA). QuaniTect Reverse Transcription Kit (QIAGEN, Germany) was used for cDNA synthesis. The 7900 HT Fast Real-Time PCR System (Applied Biosystems), Absolute QPCR SYBRGreen Mix (Thermo Scientific, UK) and pair of primers specific for each studied gene (Sigma/Aldrich, USA) were used for quantitative PCR (Table 2).

The expression of beta-actin mRNA was used as control of analyzed RNA quantity. The amplified DNA fragments were analyzed on a $2 \%$ agarose gel and visualized by $5 \mathrm{x}$-Sight DNA Stain (EUROMEDEA). An analysis of quantitative PCR was performed using special computer program "Differential expression calculator". Statistical analysis was performed as described previously [34]. All values are expressed as mean \pm SEM from five independent experiments; $\mathrm{P}<0.05$ was considered as significant difference.

\section{Results and Discussion}

We studied the expression of the subset of genes (CYR61, PLAGL, GADD45A, GADD34 (PPP1R15A), ITGA5, TFPI2,
ALDH1A2, and HSPA6), which control cell proliferation and apoptosis, in blood cells of the three groups for evaluation of its possible significance to the development of obesity and insulin resistance. As shown in Fig.1, the levels of PLAGL1 and $C Y R 61$ mRNA expression are increased $(+25$ and $+69 \%$, respectively; $P<0.05$ and $P<0.01$, respectively) in blood cells of obese boys with normal insulin sensitivity as compared to the control group.

The development of insulin resistance in obese boys is associated with additional up-regulation of PLAGL1 mRNA expression $(+34 \% ; P<0.05)$ in blood cells as compared to the group with obesity and normal insulin sensitivity. At the same time, the expression level of CYR61 mRNA is decreased $(-17 \% ; P<0.05)$ in blood cells of obese children with impaired insulin sensitivity versus the group of obese boys without insulin resistance, but it is significantly higher relative to the control group $(+41 \% ; P<0.05$; Fig. 1$)$.

These data clearly demonstrate that obesity leads to significant dysregulation of PLAGL1 and CYR61 genes in blood cells, being more evident for the CYR61 gene, and that this dysregulation of PLAGL1 and CYR61 genes possibly contributes to the development of enhanced cell proliferation, obesity, and insulin resistance. Moreover, we have also shown that development of insulin resistance in obese individuals is associated with down-regulation of CYR61 and up-regulation of PLAGL1 gene expressions. These results correlate with data

Table 2.

Characteristics of the primers used for quantitative real-time PCR

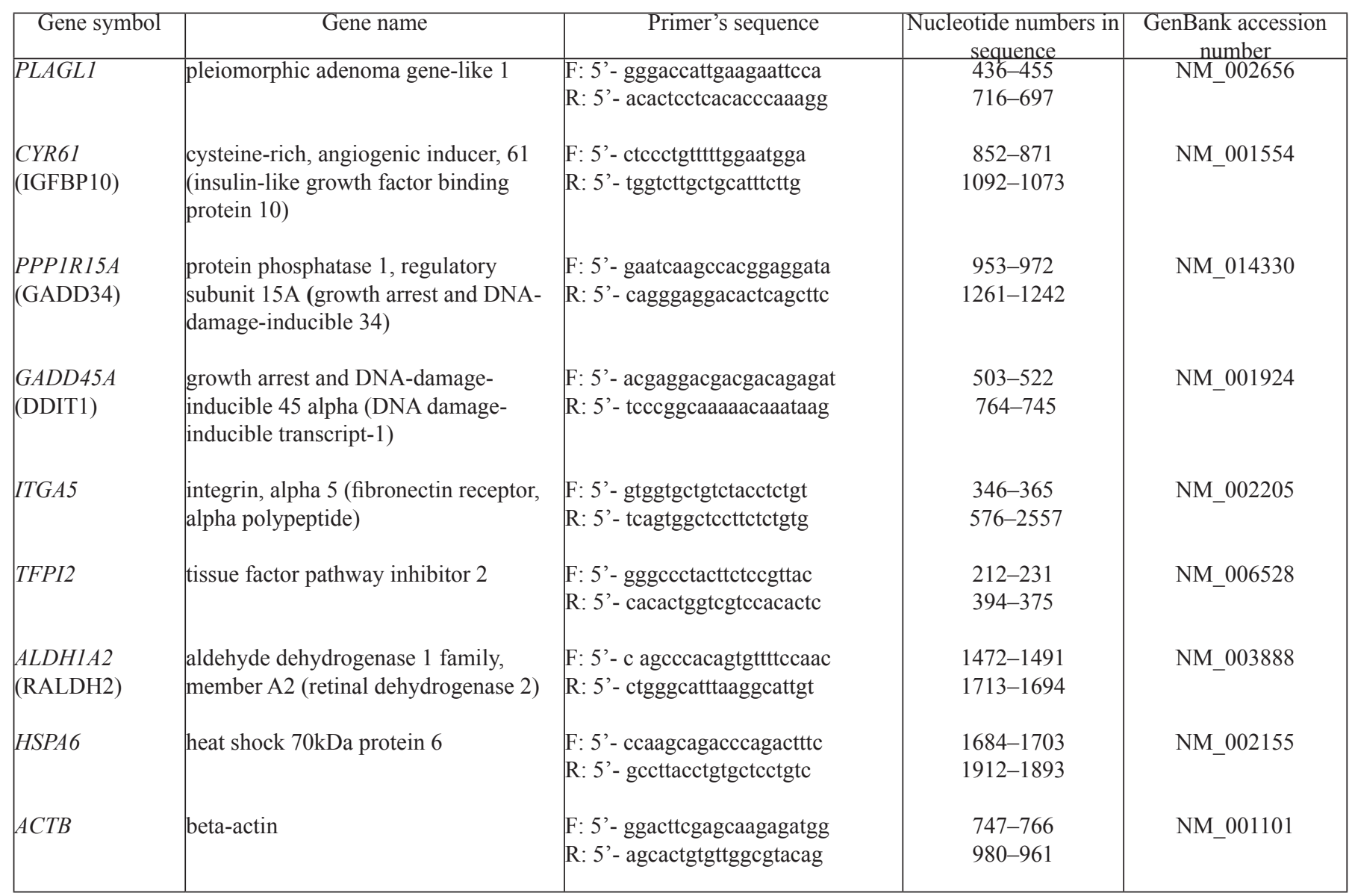


of other authors that down-regulation of CYR61 is associated with inhibition of tumor cell proliferation $[10,12]$ and that PLAGL1 may have a role in tumorigenesis of von HippelLindau-associated central nervous system hemangioblastoma [22].

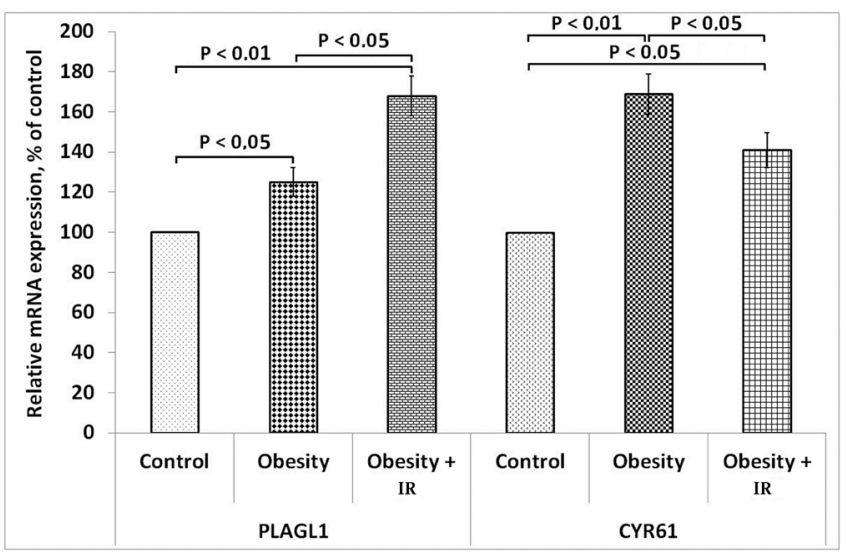

Fig.1. Relative mRNA expression of PLAGL1 and CYR61 in blood cells of normal boys (Control) and obese individuals with normal insulin sensitivity (Obesity) and obese patients with insulin resistance (Obesity $+I R)$. Levels of PLAGLI and CYR61 mRNA were normalized to the beta-actin $m R N A$ and are represented as mean $\pm S E M$ and expressed as a percent of the control (100\%).

Investigation of the expression level of the growth arrest and DNA-damage-inducible 34 (GADD34) gene, also known as regulatory subunit $15 \mathrm{~A}$ of protein phosphatase 1 (PPP1R15A), in blood cells of obese boys who have normal insulin sensitivity has revealed that obesity does not change significantly the expression of this gene when compared to the control group, but insulin resistance leads to an up-regulation of GADD34 mRNA expression $(+21 \%$; $P<0.05$; Fig. 2$)$ as compared to the group of obese boys with normal insulin sensitivity.

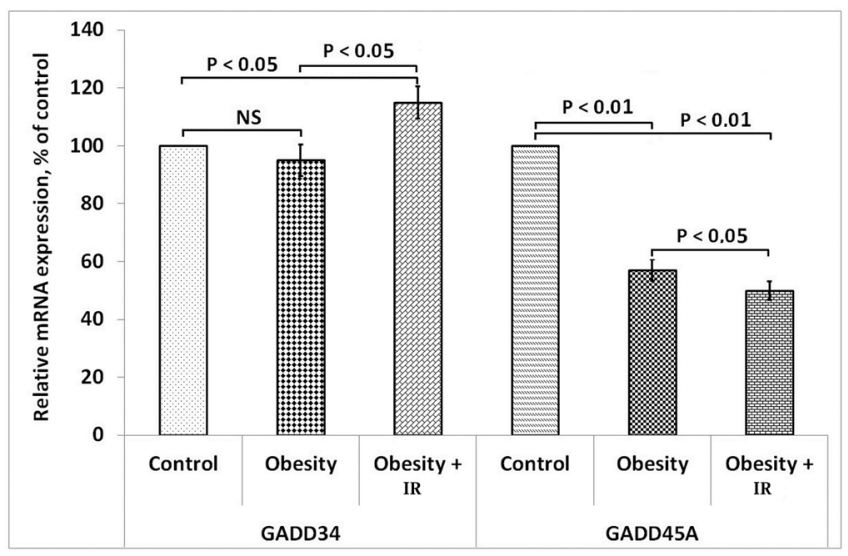

Fig. 2. Relative $m R N A$ expression of GADD34 and GADD45A in blood cells of normal boys (Control) and obese individuals with normal insulin sensitivity (Obesity) and obese patients with insulin resistance (Obesity + IR). Levels of GADD34 and GADD45A $m R N A$ were normalized to the beta-actin $m R N A$ and are represented as mean \pm SEM and expressed as a percent of the control (100\%).

At the same time, another member of the DNA-damageinducible protein family, DNA-damage-inducible proteins $45(G A D D 45 A)$, also known as DNA damage-inducible transcript-1 (DDIT1), is significantly decreased in obesity without insulin resistance (almost two fold; $P<0.05$; Fig. 2). Furthermore, development of an insulin resistance induces additional down-regulation of GADD45A mRNA expression $(-12 \% ; \mathrm{P}<0.05)$. Thus, the expression of both DNA-damageinducible proteins is decreased in obesity with normal as well as with impaired insulin sensitivity. These results completely correlate with the anti-proliferative properties of both PPPIR15A and GADD45A [15, 26, 27].

As shown in Fig.3, the expression level of both ITGA5 and TFPI 2 mRNA is increased ( +24 and $+18 \%$, respectively; $P<0.05)$ in blood cells of obese boys with normal insulin sensitivity, as compared to control children, but development of insulin resistance in obese individuals is associated with the down-regulation of TFPI2 mRNA expression $(-19 \% ; P<0.05)$ in blood cells, as compared to the group with obesity and normal insulin sensitivity, up to the level in the control boys.

At the same time, the expression level of ITGA5 mRNA does not change significantly in blood cells of obese children with impaired insulin sensitivity versus the group of obese boys without insulin resistance, and it is significantly higher relative to the control group $(+29 \%$; $P<0.05$; Fig. 3$)$.

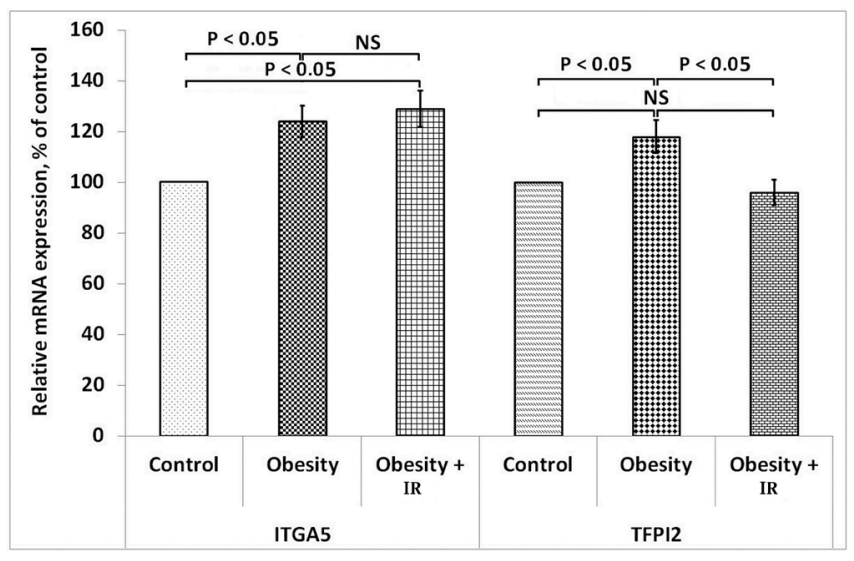

Fig.3. Relative mRNA expression of ITGA5 and TFPI2 in blood cells of normal boys (Control) and obese individuals with normal insulin sensitivity (Obesity) and obese patients with insulin resistance (Obesity + IR). Levels of ITGA5 and TFPI2 mRNA were normalized to the beta-actin $\mathrm{mRNA}$ and are represented as mean \pm SEM and expressed as a percent of the control (100\%).

The increased level of ITGA5 and TFPI2 mRNA expression agrees with the biological significance of proteins encoded by these genes, because an alpha polypeptide of fibronectin receptor as well as TFPI2 interacts with different extracellular matrix proteins, including CYR61 and vascular endothelial growth factor receptor 2, and participates in cellsurface-mediated signaling and is involved in the regulation of cell growth, division, survival, differentiation, migration and apoptosis through activation of some protein kinases $[13,14$, 23-25].

We next tested whether obesity also affects the expression of member $\mathrm{A} 2$ of the aldehyde dehydrogenase 1 family (ALDH1A2), which is a retinal dehydrogenase 2 , as well as heat shock 70kDa protein-6 (HSPAO) mRNA in blood cells of obese children with normal and impaired insulin sensitivity (Fig. 4). 


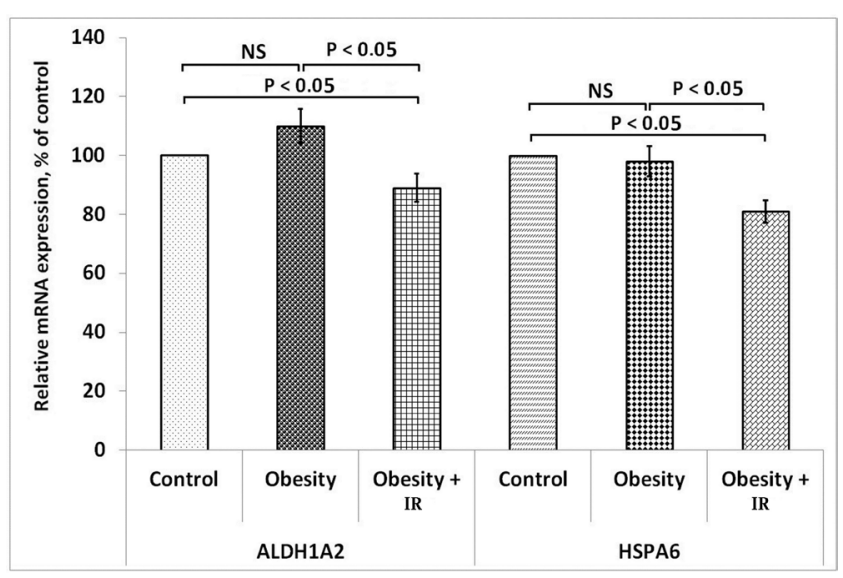

Fig.4. Relative mRNA expression of ALDH1A2 and HSPA6 in blood cells of normal boys (Control) and obese individuals with normal insulin sensitivity (Obesity) and obese patients with insulin resistance (Obesity $+I R)$. Levels of ALDH1A2 and HSPA6 $m R N A$ were normalized to the beta-actin $m R N A$ and are represented as mean \pm SEM and expressed as a percent of the control (100\%).

As shown in Fig.4, no significant changes were observed in the expression level of both ALDH1A2 and HSPA6 mRNA in blood cells of obese boys with normal insulin sensitivity, as compared to control children, but development of insulin resistance in obese individuals is associated with downregulation of both mRNA expressions (-19 and $-17 \%$, respectively; $P<0.05$ ) in blood cells versus the group with obesity and normal insulin sensitivity.

The decreased level of ALDH1A2 and HSPA6 gene expressions correlates to data that $A L D H 1 A 2$ may control the synthesis of retinoic acid, a hormonal signaling molecule that has a relation to suppression of tumor growth, and that the expression of heat shock protein A6, as a stress-responsible protein, responds to oxidized low density lipoprotein immune complexes [17, 28, 35].

Thus, results of this study provide evidence that obesity affects the expression of the subset of genes related to cellular growth and apoptosis in blood cells and that insulin resistance in obese children is associated with changes in the expression level of CYR61, PLAGL, GADD45A, PPP1R15A, TFPI2, $A L D H 1 A 2$, and HSPA6 genes, which possibly contribute to the development of obesity and its complications, and reflect some changes in other tissues, including fat tissue.

\section{Conclusions}

Obesity (with normal insulin sensitivity) enhances the expression of CYR61, ITGA5, and TFPI2 genes, which control cell growth, and decreases the expression of the GADD $45 \mathrm{~A}$ gene, which controls apoptosis, in blood cells, versus the control group. Insulin resistance in obese boys leads to upregulation of PLAGL1 and PPP1R15A gene expressions and to down-regulation of CYR61, GADD45A, ALDH1A2, TFPI2, and HSPA6 genes in the blood cells versus obese patients with normal insulin sensitivity.

This study has demonstrated that obesity affects the expression of the subset of genes related to the control of cell growth and survival in the blood and that insulin resistance in obesity is associated with changes in the expression level of
CYR61, PLAGL, GADD45A, PPP1R15A, TFPI2, ALDH1A2, and HSPA6 genes, which contribute to the development of obesity and its metabolic complications and possibly reflect some changes in other tissues.

\section{References}

1. Ozcan U, Cao Q, Yilmaz E, LeeAH, Iwakoshi NN, Ozdelen E, et al. Endoplasmic reticulum stress links obesity, insulin action, and type 2 diabetes. Science 2004; 306(5695):457-61. 2. Bray MS, Young ME. Regulation of fatty acid metabolism by cell autonomous circadian clocks: time to fatten up on information? J Biol Chem 2011; 286 (14): 11883-9.

3. Kovac J, Husse J, Oster H. A time to fast, a time to feast: the crosstalk between metabolism and the circadian clock. Mol Cells 2009; 28(2):75-80.

4. Bray MS, Young ME. The role of cell-specific circadian clocks in metabolism and disease. Obes Rev 2009; 10(Suppl 2):6-13.

5. Ruderman NB, Carling D, Prentki M, Cacicedo JM. AMPK, insulin resistance, and the metabolic syndrome. J Clin Invest 2013; 123(7):2764-72.

6. Ando H, Kumazaki M, Motosugi Y, Ushijima K, Maekawa $\mathrm{T}$, Ishikawa $\mathrm{E}$, et al. Impairment of peripheral circadian clocks precedes metabolic abnormalities in ob/ob mice. Endocrinology 2011; 152(4):1347-54.

7. Duong HA, Robles MS, Knutti D, Weitz CJ. A molecular mechanism for circadian clock negative feedback. Science 2011; 332(6036):1436-9.

8. Shimba S, Ogawa T, Hitosugi S, Ichihashi Y, Nakadaira Y, Kobayashi M, et al. Deficient of a clock gene, brain and muscle Arnt-like protein-1 (BMAL1), induces dyslipidemia and ectopic fat formation. PLoS One 2011; 6(9):e25231.

9. Wang S, Kaufman RJ. The impact of the unfolded protein response on human disease. J Cell Biol 2012; 197(7):857-67. 10. Huang J, Gao K, Lin J, Wang Q. MicroRNA-100 inhibits osteosarcoma cell proliferation by targeting Cyr61. Tumour Biol 2014; 35(2):1095-100.

11. Long QZ, Zhou M, Liu XG, Du YF, Fan JH, Li X, et al. Interaction of $\mathrm{CCN} 1$ with $\alpha v \beta 3$ integrin induces $\mathrm{P}$-glycoprotein and confers vinblastine resistance in renal carcinoma cells. Anticancer Drugs 2013; 24(8):810-7.

12. Vieria Neto L, Wildemberg LE, Colli LM, Kasuki L, Marques NV, Moraes AB, et al. ZAC1 and SSTR2 are downregulated in non-functioning pituitary adenomas but not in somatotropinomas. PLoS ONE 2013; 8(10):e77406.

13. Caffarel MM, Chattopadhyay A, Araujo AM, Bauer J, Scarpini CG, Coleman N. Tissue transglutaminase mediates the pro-malignant effects of oncostatin $\mathrm{M}$ receptor overexpression in cervical squamous cell carcinoma. J Pathol 2013; 231(2):168-79.

14. Arakawa N, Miyagi E, Nomura A, Morita E, Ino Y, Ohtake N, et al. Secretome-based identification of TFPI2, a novel serum biomarker for detection of ovarian clear cell adenocarcinoma. J Proteome Res 2013; 12(10):4340-50.

15. Hoffman B, Liebermann DA. Gadd45 in modulation of solid tumors and leukemia. Adv Exp Med Biol 2013; 793:21-33. 16. Zhou W, Jeyaraman K, Yusoff P, Shenolikar S. Phosphorylation at tyrosine 262 promotes GADD34 protein turnover. J Biol Chem 2013; 288(46):33146-55.

17. Kim H, Lapointe J, Kaygusuz G, Ong DE, Li C, van de Rijn $\mathrm{M}$, et al. The retinoic acid synthesis gene ALDH1a2 is a candidate tumor suppressor in prostate cancer. Cancer Res 
2005; 65(18):8118-24.

18. Lin J, Huo R, Xiao L, Zhu X, Xie J, Sun S, et al. A novel p53/microRNA-22/Cyr61 axis in synovial cells regulates inflammation in rheumatoid arthritis. Arthritis Rheumatol 2014; 66(1):49-59.

19. Lee YJ, Lee DM, Lee SH. Production of Cyr61 protein is modulated by extracellular acidification and PI3K/Akt signaling in prostate carcinoma PC-3 cells. Food Chem Toxicol 2013; 58:169-76.

20. Choi J, Lin A, Shrier E, Lau LF, Grant MB, Chaqour B. Degradome products of the matricellular protein $\mathrm{CCN} 1$ as modulators of pathological angiogenesis in the retina. J Biol Chem 2013; 288(32):23075-89.

21. Du X, Rousseau M, Ounissi-Benkalha H, Marchand L, Jetha A, Paraskevas S, et al. Differential expression pattern of ZAC in developing mouse and human pancreas. J Mol Histol 2011; 42(2):129-36.

22. Zhou J, Wang J, Li N, Zhang X, Zhou H, Zhang R, et al. Molecularly genetic analysis of von Hippel-Lindau associated central nervous system hemangioblastoma. Pathol Int 2010; 60(6):452-8.

23. Walter RB, Laszlo GS, Alonzo TA, Gerbing RB, Levy S, Fitzgibbon MP, et al. Significance of expression of ITGA5 and its splice variants in acute myeloid leukemia: a report from the Children's Oncology Group. Am J Hematol 2013; 88(8):694702.

24. Xu C, Wang H, He H, Zheng F, Chen Y, Zhang J, et al. Low expression of TFPI-2 associated with poor survival outcome in patients with breast cancer. BMC Cancer 2013; $13: 118$.

25. Takada H, Wakabayashi N, Dohi O, Yasui K, Sakakura C, Mitsufuji S, et al. Tissue factor pathway inhibitor 2 (TFPI2) is frequently silenced by aberrant promoter hypermethylation in gastric cancer. Cancer Genet Cytogenet 2010; 197(1):16-24. 26. Salvador JM, Brown-Clay JD, Fornace AJ Jr. Gadd45 in stress signaling, cell cycle control, and apoptosis. Adv Exp Med Biol 2013; 793:1-19.
27. Dalton LE, Clarke HJ, Knight J, Lawson MH, Wason J, Lomas DA, et al. The endoplasmic reticulum stress marker CHOP predicts survival in malignant mesothelioma. $\mathrm{Br} \mathrm{J}$ Cancer 2013; 108(6):1340-7.

28. Gyongyosi A, Szatmari I, Pap A, Dezso B, Pos Z, Szeles L, et al. RDH10, RALDH2, and CRABP2 are required components of PPAR $\gamma$-directed ATRA synthesis and signaling in human dendritic cells. J Lipid Res 2013; 54(9):2458-74. 29. Han S, Lan Q, Park AK, Lee KM, Park SK, Ahn HS, et al. Polymorphisms in innate immunity genes and risk of childhood leukemia. Hum Immunol 2010; 71(7):727-30.

30. Lee J, Ozcan U. Unfolded protein response signaling and metabolic diseases. J Biol Chem 2014; 289(3):1203-11.

31. Kryvdiuk I, Bakalets T, Riabovol O, Minchenko D. Expression of GADD34 and GADD45 mRNA in U87 glioma cells: effect of blockade of ERN1 signaling enzyme and glucose deprivation. Visnyk Taras Shevchenko Kyiv National Univ (Biology) 2014; 66 (1): 28-32.

32. Yuzefovych LV, Musiyenko SI, Wilson GL, Rachek LI. Mitochondrial DNA damage and dysfunction, and oxidative stress are associated with endoplasmic reticulum stress, protein degradation and apoptosis in high fat diet-induced insulin resistance mice. PLoS One 2013; 8(1):e54059.

33. Minchenko D, Ratushna O, Bashta Y, Herasymenko R, Minchenko O. The expression of TIMP1, TIMP2, VCAN, SPARC, CLEC3B and E2F1 in subcutaneous adipose tissue of obese males and glucose intolerance. CellBio 2013; 2(2):25-33. 34. Bochkov VN, Philippova M, Oskolkova O, Kadl A, Furnkranz A, Karabeg E, et al. Oxidized phospholipids stimulate angiogenesis via autocrine mechanisms, implicating a novel role for lipid oxidation in the evolution of atherosclerotic lesions. Circ Res 2006; 99(8):900-8.

35. Smith KJ, Twal WO, Soodavar F, Virella G, Lopes-Virella MF, Hammad SM. Heat shock protein 70B' (HSP70B') expression and release in response to human oxidized low density lipoprotein immune complexes in macrophages. J Biol Chem 2010; 285(21):15985-93. 